\title{
Solution of fractional oxygen diffusion problem having without singular kernel
}

\author{
Badr S. Alkahtani ${ }^{\mathrm{a}}$, Obaid J. Algahtani ${ }^{\mathrm{a}}$, Ravi Shanker Dubey ${ }^{\mathrm{b}}$, Pranay Goswami ${ }^{\mathrm{c}, *}$ \\ a Mathematics Department, College of Science, King Saud University, Riyadh 11989, Saudi Arabia. \\ ${ }^{b}$ Department of Mathematics, Yagyavalkya Institute of Technology, Jaipur-302022, India. \\ ${ }^{c}$ School of Liberal Studies, Ambedkar University Delhi, Delhi-11006, India.
}

Communicated by A. Atangana

\begin{abstract}
In the present paper, we use an efficient approach to solve fractional differential equation, oxygen diffusion problem which is used to describe oxygen absorption in human body. The oxygen diffusion problem is considered in new Caputo derivative of fractional order in this paper. Using an iterative approach, we derive the solutions of the modified system. (C)2017 all rights reserved.
\end{abstract}

Keywords: Oxygen diffusion problem, Caputo-Fabrizio fractional derivative, fractional differential equation, Laplace transform, fixed-point theorem.

2010 MSC: 26A33, 35A22, 33E12, 35R11, 65L10.

\section{Introduction and preliminaries}

The distribution of oxygen into absorbing tissue was first studied by Crank and Gupta [11]. When the oxygen is allowed to diffuse into a medium, some part of the oxygen is absorbed by the medium and absorption of oxygen at the surface of the medium is maintained constant. This phase of the problem continues until a steady state is achieved in which the oxygen does not penetrate any further so that no oxygen passes in or out, the medium continues to absorb the available oxygen already in it and, as a consequence, the boundary in the steady state starts to recede towards the sealed surface. Crank and Gupta [10] also employed a uniform space grid moving with the boundary and necessary interpolations are performed with either cube splines or polynomials. In this direction Noble suggested the repeated spatial subdivision [20], the heat balance integral method defined by Reynolds and Dalton [22], an orthogonal collocation for solving the partial differential equation of the diffusion of oxygen in absorbing tissue described by Liapis et al. [16]. Two numerical methods for solving the oxygen diffusion problem were proposed by Gülkaç [14]. Mitchell studied the accurate application of the integral method [19]. For more details see $[2,5,6,8,9,13,15,18,21]$.

\footnotetext{
${ }^{*}$ Corresponding author

Email addresses: alhaghog@gmail.com (Badr S. Alkahtani), obalgahtani@ksu.edu .sa (Obaid J. Algahtani), ravimath13@gmail.com (Ravi Shanker Dubey), pranaygoswami83@gmail.com (Pranay Goswami)

doi:10.22436/jnsa.010.01.28
} 
In applied mathematics, one of the most used concepts is derivative. Derivative shows the rate of change of the function. This is also helpful to describe many real phenomena. After this research, the mathematicians faced some complex problems of real world. To solve them mathematicians introduced fractional derivative (see $[2,6,8,15,21])$. The concept of fractional calculus has great importance in many branches and is also important for modeling real world problem (see $[5,9,13])$.

Due to this region a lot of research work, conference, and paper publication have been done by many researchers. In this concern varies definitions of fractional derivative have been given till now. Recently the researchers described the new fractional derivative operator named Caputo-Fabrizio fractional derivative (see $[3,4,7,12,17])$.

\subsection{The Caputo-Fabrizio fractional order derivative}

Singularity at the end point of the interval is the main problem which is faced with the definition of fractional order derivative. To avoid this problem, Caputo-Fabrizio recently proposed a new fractional order derivative which does not have any singularity. The definition is based on the convolution of a first order derivative and the exponential function, given in the following definition:

Definition 1.1. Let $f \in H^{1}(a, b), b>a, \alpha \in[0,1]$, then the new fractional order Caputo derivative is defined as:

$$
D_{t}^{\alpha}(f(t))=\frac{M(\alpha)}{(1-\alpha)} \int_{a}^{t} f^{\prime}(x) e^{\left[-\alpha \frac{t-x}{1-\alpha}\right]} d x
$$

Here $M(\alpha)$ denotes the normalization function such as $M(0)=M(1)=1$, for details see [7].

As we have defined a new derivative, then there should be its anti-derivative, the integral of this new fractional derivative is given by Losada and Nieto [17].

Definition 1.2. The fractional integral of order $\alpha(0<\alpha<1)$, of the function $\mathrm{f}$ is defined as below

$$
I_{\alpha}^{t}(f(t))=\frac{2(1-\alpha)}{(2-\alpha) M(\alpha)} f(t)+\frac{2 \alpha}{(2-\alpha) M(\alpha)} \int_{0}^{t} f(s) d s, t \geqslant 0 .
$$

\subsection{Laplace transform}

One of the simplest and most important integral transforms which has been a subject of wide and extensive study by authors due to its various uses in applied mathematics, is well-known Laplace transform defined as follows

$$
\begin{aligned}
\mathrm{L}(\mathrm{f}(\mathrm{t})) & =\mathrm{F}(\mathrm{s}), \\
\mathrm{L}\{\mathrm{f}(\mathrm{t}) ; \mathrm{s}\} & =\int_{0}^{\infty} \mathrm{e}^{-\mathrm{st}} \mathrm{f}(\mathrm{t}) \mathrm{dt} .
\end{aligned}
$$

The Laplace transform of the Caputo-Fabrizio fractional order derivative is given as [1], defined below

$$
L\left(\left(\begin{array}{ll}
C F \\
0
\end{array} D_{t}^{\alpha}\right)(f(t))\right)=\left[\frac{s L(f(t))-f(0)}{s+\alpha(1-s)}\right]
$$

\subsection{Oxygen diffusion problem fractional module}

The model of oxygen diffusion problem is given by Crank and Gupta [11]. The oxygen diffusion problem has two mathematical stages. At the first stage, the stable condition occurs when the oxygen is injected into either from the inside or outside of the cell then the cell surface is isolated.

At the second stage, tissues start to absorb the injected oxygen. The moving boundary problem is caused by this level. The aim of this process is to find a balance position and to determine the timedependent moving boundary position. For detail of time-fractional of oxygen diffusion problem see $[11,19]$. 
We consider the following oxygen diffusion problem

$$
\left(\begin{array}{l}
C F \\
0
\end{array} D_{t}^{\alpha}\right)(c(x, t))=c_{x x}-1 ; \quad x, t \in \phi,
$$

with the following initial and boundary conditions

$$
\begin{gathered}
c(x, 0)=\frac{(1-x)^{2}}{2}, 0 \leqslant x \leqslant 1, \\
\frac{\partial c}{\partial x}=0, x=0, t \geqslant 0 .
\end{gathered}
$$

\section{Existence of the coupled solutions}

By using the fixed-point theorem and following the procedure described in [1], we define the existence of the solution. First, we transform equation (1.1) to an integral equation as follows

$$
c(x, t)-c(x, 0)={ }_{0}^{C F} I_{t}^{\alpha}\left[c_{x x}-1\right],
$$

by using the definition defined by Nieto, we get

$$
c(x, t)=c(x, 0)+\frac{2(1-\alpha)}{(2-\alpha) M(\alpha)}\left\{\frac{\partial^{2} c(x, t)}{\partial x^{2}}-1\right\}+\frac{2 \alpha}{(2-\alpha) M(\alpha)} \int_{0}^{t}\left[\frac{\partial^{2} c(x, s)}{\partial x^{2}}-1\right] d s
$$

Let us consider the following kernels

$$
K_{1}(x, t, c)=\frac{\partial^{2} c(x, t)}{\partial x^{2}}-1
$$

Theorem 2.1. $\mathrm{K}_{1}$ satisfies Lipschitz condition and contraction if the following inequality holds

$$
0 \leqslant \delta^{2} \leqslant 1
$$

Proof. Let $\mathrm{c}$ and $\mathrm{c}_{1}$ be two functions, then we have

$$
\left\|K_{1}(x, t, c)-K_{1}\left(x, t, c_{1}\right)\right\|=\left\|\frac{\partial^{2} c(x, t)}{\partial x^{2}}-\frac{\partial^{2} c_{1}(x, t)}{\partial x^{2}}\right\| .
$$

Since we know such as the operator derivative satisfies the Lipschitz condition, then we can find positive parameter $\delta$ such that

$$
\left\|\frac{\partial^{2} c(x, t)}{\partial x^{2}}-\frac{\partial^{2} c_{1}(x, t)}{\partial x^{2}}\right\| \leqslant \delta^{2}\left\|\left(c(x, t)-c_{1}(x, t)\right)\right\| .
$$

Putting the value from Eq. (2.2) into Eq. (2.1), we obtain

$$
\left\|K_{1}(x, t, c)-K_{1}\left(x, t, c_{1}\right)\right\| \leqslant \delta^{2}\left\|\left(c(x, t)-c_{1}(x, t)\right)\right\| .
$$

Consider $\delta^{2}=A$, then we get

$$
\left\|K_{1}(x, t, c)-K_{1}\left(x, t, c_{1}\right)\right\| \leqslant A\left\|\left(c(x, t)-c_{1}(x, t)\right)\right\| .
$$

Therefore $K_{1}$ satisfies the Lipschitz conditions and if in addition $0 \leqslant \delta^{2} \leqslant 1$, then it is also a contraction.

We consider the following recursive formula

$$
c_{n}(x, t)=c(x, 0)+\frac{2(1-\alpha)}{(2-\alpha) M(\alpha)} K_{1}\left(x, t, c_{n-1}\right)+\frac{2 \alpha}{(2-\alpha) M(\alpha)} \int_{0}^{t}\left\{K_{1}\left(x, t, c_{n-1}\right)\right\} d s,
$$


with initial component

$$
c_{0}(x, t)=c(x, 0) .
$$

Now the difference between the consecutive terms is

$$
\begin{aligned}
\mathrm{U}_{n}(x, t)=\mathrm{c}_{n}(x, t)-\mathrm{c}_{n-1}(x, t)= & \frac{2(1-\alpha)}{(2-\alpha) M(\alpha)} \mathrm{K}_{1}\left(x, t, c_{n-1}\right)-\frac{2(1-\alpha)}{(2-\alpha) M(\alpha)} K_{1}\left(x, t, c_{n-2}\right) \\
& +\frac{2 \alpha}{(2-\alpha) M(\alpha)} \int_{0}^{t}\left\{K_{1}\left(x, s, c_{n-1}\right)-K_{1}\left(x, s, c_{n-2}\right)\right\} d s,
\end{aligned}
$$

here

$$
c_{n}(x, t)=\sum_{n=0}^{\infty} u_{n}(x, t) .
$$

Now take norm on both sides of equation (2.4), we get

$$
\begin{aligned}
\left\|\mathrm{U}_{\mathrm{n}}(x, \mathrm{t})\right\|= & \left\|\mathrm{c}_{n}(x, \mathrm{t})-\mathrm{c}_{n-1}(x, \mathrm{t})\right\| \\
= & \| \frac{2(1-\alpha)}{(2-\alpha) M(\alpha)} \mathrm{K}_{1}\left(x, s, \mathrm{c}_{n-1}\right)-\frac{2(1-\alpha)}{(2-\alpha) M(\alpha)} \mathrm{K}_{1}\left(x, t, c_{n-2}\right) \\
& +\frac{2 \alpha}{(2-\alpha) M(\alpha)} \int_{0}^{t}\left\{K_{1}\left(x, s, c_{n-1}\right)-K_{1}\left(x, s, c_{n-2}\right)\right\} d s \| .
\end{aligned}
$$

From Eq. (2.5) we can say that

$$
\begin{aligned}
\left\|\mathrm{U}_{n}(x, t)\right\|= & \left\|c_{n}(x, t)-c_{n-1}(x, t)\right\| \\
\leqslant & \frac{2(1-\alpha)}{(2-\alpha) M(\alpha)}\left\|K_{1}\left(x, s, c_{n-1}\right)-K_{1}\left(x, t, c_{n-2}\right)\right\| \\
& +\frac{2 \alpha}{(2-\alpha) M(\alpha)}\left\|\int_{0}^{t}\left\{K_{1}\left(x, s, c_{n-1}\right)-K_{1}\left(x, t, c_{n-2}\right)\right\}\right\| d s .
\end{aligned}
$$

Since by the above discussion we have seen that kernel satisfies the Lipschitz condition, so we get

$$
\begin{aligned}
\left\|c_{n}(x, t)-c_{n-1}(x, t)\right\| \leqslant & \frac{2(1-\alpha)}{(2-\alpha) M(\alpha)} A\left\|c_{n-1}(x, t)-c_{n-2}(x, t)\right\| \\
& +\frac{2 \alpha}{(2-\alpha) M(\alpha)} B \int_{0}^{t}\left\{\left\|c_{n-1}(x, s)-c_{n-2}(x, s)\right\|\right\} d s
\end{aligned}
$$

or

$$
\left\|\mathrm{U}_{\mathrm{n}}(x, \mathrm{t})\right\| \leqslant \frac{2(1-\alpha)}{(2-\alpha) M(\alpha)} \mathrm{A}\left\|\mathrm{U}_{\mathrm{n}-1}(x, \mathrm{t})\right\|+\frac{2 \alpha}{(2-\alpha) M(\alpha)} B \int_{0}^{t}\left\{\left\|\mathrm{U}_{n-1}(x, s)\right\|\right\} \mathrm{ds}
$$

Theorem 2.2. Oxygen diffusion problem fractional module is the model of the oxygen absorption in human body having a solution.

Proof. As we have seen, the above equation (2.2), is bounded, as well as, we have proved that the kernel satisfies the Lipschitz condition, therefore the following results obtained in equation (2.3) using the recursive technique, we get the following relation

$$
\left\|\mathrm{u}_{\mathrm{n}}(\mathrm{x}, \mathrm{t})\right\| \leqslant\|\mathrm{u}(\mathrm{x}, 0)\|\left\{\left(\frac{2(1-\alpha)}{(2-\alpha) M(\alpha)} \mathrm{A}\right)^{\mathrm{n}}+\left(\frac{2 \alpha}{(2-\alpha) M(\alpha)} \mathrm{Bt}\right)^{\mathrm{n}}\right\} .
$$


Therefore, the above solutions exist and are continuous. Nonetheless, to show that the above is a solution of Eq. (1.1), we get

$$
c(x, t)=c_{n}(x, t)-P_{n}(x, t)
$$

Thus

$$
c(x, t)-c_{n}(x, t)=\frac{2(1-\alpha)}{(2-\alpha) M(\alpha)} K_{1}\left(x, t, c-P_{n}(x, s)\right)+\frac{2 \alpha}{(2-\alpha) M(\alpha)} \int_{0}^{t} K_{1}\left(x, s, c-P_{n}(x, s)\right) d s .
$$

It follows from the above that

$$
\begin{aligned}
& c(x, t)-\frac{2(1-\alpha)}{(2-\alpha) M(\alpha)} K_{1}(x, t, c)-c(x, 0)-\frac{2 \alpha}{(2-\alpha) M(\alpha)} \int_{0}^{t} K_{1}(x, s, c) d s \\
& \left.\quad=P_{n}(x, t)+\frac{2(1-\alpha)}{(2-\alpha) M(\alpha)} K_{1}(x, t, c)+\frac{2 \alpha}{(2-\alpha) M(\alpha)} \int_{0}^{t}\left\{K_{1}(x, s, c)-P_{n}(x, s)\right)-K_{1}(s, x, c)\right\} d s .
\end{aligned}
$$

Now apply the norm on both sides and using the Lipchitz condition, we get

$$
\begin{aligned}
& \left\|c(x, t)-\frac{2(1-\alpha)}{(2-\alpha) M(\alpha)} K_{1}(x, t, c)-c(x, 0)-\frac{2 \alpha}{(2-\alpha) M(\alpha)} \int_{0}^{t} K_{1}(x, s, c) d s\right\| \\
& \leqslant\left\|P_{n}(x, t)\right\|+\left\{\frac{2(1-\alpha)}{(2-\alpha) M(\alpha)} A+\frac{2 \alpha}{(2-\alpha) M(\alpha)} B t\right\}\left\|P_{n}(x, t)\right\| .
\end{aligned}
$$

By taking limit $n \rightarrow \infty$ of equation (2.6), we get

$$
c(x, t)=\frac{2(1-\alpha)}{(2-\alpha) M(\alpha)} K_{1}(x, t, c)+c(x, 0)+\frac{2 \alpha}{(2-\alpha) M(\alpha)} \int_{0}^{t} K_{1}(x, s, c) d s .
$$

Eq. (2.7) is the solution of the Eq. (1.1), hence we can say that solution exists.

\section{Uniqueness of the solution}

Now in this part, we want to show that the solution presented in Section 2 is unique.

To prove this, we consider that we can find another solution for system (1.1), say $c(x, t)$ then

$$
\begin{aligned}
c(x, t)-c_{1}(x, t)= & \frac{2(1-\alpha)}{(2-\alpha) M(\alpha)}\left\{K_{1}(x, t, c)-K_{1}\left(x, t, c_{1}\right)\right\} \\
& +\frac{2 \alpha}{(2-\alpha) M(\alpha)} \int_{0}^{t}\left\{K_{1}(x, s, c)-K_{1}\left(x, s, c_{1}\right)\right\} d s .
\end{aligned}
$$

Apply the norm on the both sides of equation (3.1),

$$
\begin{aligned}
\left\|c(x, t)-c_{1}(x, t)\right\| \leqslant & \frac{2(1-\alpha)}{(2-\alpha) M(\alpha)}\left\{\left\|K_{1}(x, t, c)-K_{1}\left(x, t, c_{1}\right)\right\|\right\} \\
& +\frac{2 \alpha}{(2-\alpha) M(\alpha)} \int_{0}^{t}\left\{\left\|K_{1}(x, s, c)-K_{1}\left(x, s, c_{1}\right)\right\|\right\} d s .
\end{aligned}
$$

By using the Lipchitz condition, having the fact in mind that the solution is bounded, we get

$$
\left\|c(x, t)-c_{1}(x, t)\right\|<\frac{2(1-\alpha)}{(2-\alpha) M(\alpha)} H D+\left\{\frac{2 \alpha}{(2-\alpha) M(\alpha)}\left(J_{1} D t\right)\right\}^{n},
$$

this is true for any $n$, hence

$$
c(x, t)=c_{1}(x, t)
$$

Hence it shows the uniqueness of the solution of system (1.1). 


\section{Analysis of Laplace transform homotopy perturbation method (LTHPM) with time-fractional deriva- tives}

Let us consider, the nonlinear fractional differential equation is as follows

$$
{ }_{0}^{C F} D_{t}^{\alpha} u(x, t)=\mathfrak{I}(u)+N(u)+f(x, t), x, t \in \Omega
$$

with the following initial condition $u(x, 0)=\phi$, where $\mathfrak{I}$ is the linear operator and $N$ is the non-linear operator and $f$ is known function.

For the solution by LTHPM we use steps of standard HPM; next we apply Laplace transform on both sides of homotopy equation, we get

$$
H(U, p)=(1-p)\left[{ }_{0}^{C F} D_{t}^{\alpha} U-u_{0}\right]+p\left[{ }_{0}^{C F} D_{t}^{\alpha} U-\mathfrak{I}(U)-N(U)-f(x, t)\right]=0
$$

or

$$
H(U, p)={ }_{0}^{C F} D_{t}^{\alpha} U-u_{0}+p\left[u_{0}-\mathfrak{I}(U)-N(U)-f(x, t)\right]=0,
$$

where $p \in[0,1]$ and $u_{0}$ is the initial approximation for the solution of Eq. (4.1).

Clearly, the homotopy equations $H(U, 0)=0$ and $H(U, 1)=1$ are equivalent to the equations ${ }_{0}^{C F} D_{t}^{\alpha} U-u_{0}=0$ and $\left[{ }_{0}^{C F} D_{t}^{\alpha} U-\mathfrak{I}(U)-N(U)-f(x, t)\right]=0$, respectively. Thus, a monotonous change of parameter $\mathrm{p}$ from 0 to 1 corresponds to a continuous change of the trivial problem ${ }_{0}^{C F} \mathrm{D}_{\mathrm{t}}^{\alpha} \mathrm{U}-\mathrm{u}_{0}=0$ to the original problem. Now, we assume that the solution of Eq. (4.1) can be written as a power series in embedding parameter $\mathrm{p}$, as follows

$$
\mathrm{U}=\mathrm{U}_{0}+\mathrm{pu}_{1},
$$

where $\mathrm{U}_{0}$ and $\mathrm{U}_{1}$ are functions which should be determined. Now, we can write Eq. (4.2) in the following form

$$
{ }_{0}^{C F} D_{t}^{\alpha} U=u_{0}+p\left[-u_{0}+\mathfrak{I}(U)+N(U)+f(x, t)\right] .
$$

Applying the Laplace transform on both sides of Eq. (4.2), we have

$$
\begin{aligned}
& \mathrm{L}\left\{\begin{array}{l}
\mathrm{CF} \\
0
\end{array} \mathrm{D}_{\mathrm{t}}^{\alpha} \mathrm{U}\right\}=\mathrm{L}\left\{\mathrm{u}_{0}+\mathrm{p}\left[-\mathrm{u}_{0}+\mathfrak{I}(\mathrm{U})+\mathrm{N}(\mathrm{U})+\mathrm{f}(\mathrm{x}, \mathrm{t})\right]\right\}, \\
& \frac{s^{n+1} L(U)-s^{n} U(0)-s^{n-1} U^{\prime}(0)-\cdots-U^{n}(0)}{s+\alpha(1-s)}=L\left\{u_{0}+p\left[-u_{0}+\mathfrak{I}(U)+N(U)+f(x, t)\right]\right\},
\end{aligned}
$$

or

$$
\begin{aligned}
L(U)= & \frac{s^{n} U(0)+s^{n-1} U^{\prime}(0)+\ldots+U^{n}(0)}{s^{n+1}} \\
& +\{s+\alpha(1-s)\}\left[L\left\{u_{0}+p\left[-u_{0}+\mathfrak{I}(U)+N(U)+f(x, t)\right]\right\}\right] .
\end{aligned}
$$

By tacking inverse Laplace transform of Eq. (4.3), we get

$$
\begin{aligned}
U= & L^{-1}\left\{\left(\frac{s^{n} U(0)+s^{n-1} U^{\prime}(0)+\ldots+U^{n}(0)}{s^{n+1}}\right.\right. \\
& \left.\left.+\{s+\alpha(1-s)\}\left[L\left\{u_{0}+p\left[-u_{0}+\mathfrak{I}(U)+N(U)+f(x, t)\right]\right\}\right]\right)\right\} .
\end{aligned}
$$

Suppose that the initial approximation of the solutions of Eq. (4.1) are in the following form

$$
u_{0}=\sum_{k=0}^{\infty} a_{k}(x) \frac{t^{\alpha k}}{\Gamma(\alpha k+1)},
$$

where $a_{k}(x)$ for $k=1,2, \cdots$ are functions which must be computed. 
Comparing coefficients of $p$ with the same power leads to

$$
p^{0}: U_{0}=L^{-1}\left(\frac{s^{n} U(0)+s^{n-1} U^{\prime}(0)+\cdots+U^{n}(0)}{s^{n+1}}+\frac{\{s+\alpha(1-s)\}}{s^{n+1}}\left[L\left(u_{0}\right)\right]\right)
$$

and

$$
\mathrm{p}^{1}: \mathrm{U}_{1}=\mathrm{L}^{-1}\left(\frac{\{\mathrm{s}+\alpha(1-\mathrm{s})\}}{\mathrm{s}^{\mathrm{n}+1}}\left\{\mathrm{~L}\left[-\mathrm{u}_{0}+\mathfrak{I}(\mathrm{U})+\mathrm{N}(\mathrm{U})+\mathrm{f}(\mathrm{x}, \mathrm{t})\right]\right\}\right) .
$$

Putting the values from Eqs. (4.4) and (4.5) in Eq. (4.2), as $p \rightarrow 1$, we get the required solution

$$
\mathrm{U}=\mathrm{U}_{0}+\mathrm{U}_{1} \text {. }
$$

\section{Solution of fractional oxygen diffusion problem by LTHPM}

Consider the following oxygen diffusion problem

$$
\left({ }_{0}^{C F} D_{t}^{\alpha}\right)(c(x, t))=c_{x x}-1 ; \quad x, t \in \phi
$$

with the following initial and boundary conditions

$$
c(x, 0)=\frac{(1-x)^{2}}{2}, 0 \leqslant x \leqslant 1 .
$$

The objective of this section is to employ LTHPM to find analytical approximate solutions of nonlinear fractional differential equation (5.1).

For this purpose LTHPM follows the same steps of standard HPM; next we apply Laplace transform on both sides of homotopy equation, then we get,

$$
\mathrm{L}\left\{0_{0}^{C F} D_{t}^{\alpha} \mathrm{U}-\mathrm{u}_{0}+\mathrm{p}\left[\mathrm{u}_{0}-\mathrm{I}(\mathrm{U})-\mathrm{N}(\mathrm{U})-\mathrm{f}(\mathrm{x}, \mathrm{t})\right]\right\}=0 .
$$

We assume that the initial approximation of the solutions of Eq. (4.1) are in the following form

$$
\mathrm{u}_{0}=\sum_{\mathrm{k}=0}^{\infty} \mathrm{a}_{\mathrm{k}}(\mathrm{x}) \frac{\mathrm{t}^{\alpha \mathrm{k}}}{\Gamma(\alpha \mathrm{k}+1)}
$$

where $a_{k}(x)$ for $k=1,2, \cdots$ are functions which must be computed.

$$
\frac{s(c(x, s))-c(x, 0)}{s+\alpha(1-s)}=L\left\{\left[\sum_{k=0}^{\infty} a_{k}(x) \frac{t^{\alpha k}}{\Gamma(\alpha k+1)}\right]-p\left[\sum_{k=0}^{\infty} a_{k}(x) \frac{t^{\alpha k}}{\Gamma(\alpha k+1)}+c_{x x}-1\right]\right\},
$$

or

$$
c(x, s)=\frac{(1-x)^{2}}{2 s}+\left(\frac{s+\alpha(1-s)}{s}\right) L\left\{\left[\sum_{k=0}^{\infty} a_{k}(x) \frac{t^{\alpha k}}{\Gamma(\alpha k+1)}\right]-p\left[\sum_{k=0}^{\infty} a_{k}(x) \frac{t^{\alpha k}}{\Gamma(\alpha k+1)}+c_{x x}-1\right]\right\} .
$$

By tacking the inverse Laplace transform, we get

$$
\begin{aligned}
c(x, t)= & L^{-1}\left[\frac{(1-x)^{2}}{2 s}\right. \\
& \left.+\left(\frac{s+\alpha(1-s)}{s}\right) L\left\{\left[\sum_{k=0}^{\infty} a_{k}(x) \frac{t^{\alpha k}}{\Gamma(\alpha k+1)}\right]-p\left[\sum_{k=0}^{\infty} a_{k}(x) \frac{t^{\alpha k}}{\Gamma(\alpha k+1)}+c_{x x}-1\right]\right\}\right] .
\end{aligned}
$$

Collecting the same powers of $p$, and equating each coefficients of $p$ to zero yields

$$
p^{0}: c_{0}(x, t)=\frac{(1-x)^{2}}{2}+L^{-1}\left[\left(\frac{s+\alpha(1-s)}{s}\right)\left\{L\left[\sum_{k=0}^{\infty} a_{k}(x) \frac{t^{\alpha k}}{\Gamma(\alpha k+1)}\right]\right\}\right]
$$


and

$$
p^{1}: c_{1}(x, t)=L^{-1}\left[\left(\frac{s+\alpha(1-s)}{s}\right)\left\{L\left[-\sum_{k=0}^{\infty} a_{k}(x) \frac{t^{\alpha k}}{\Gamma(\alpha k+1)}+c_{0 x x}-1\right]\right\}\right],
$$

where

$$
\mathrm{c}_{0 x x}(x, t)=\sum_{k=0}^{\infty} \frac{t^{\alpha k}}{\Gamma(\alpha k+1)}
$$

and also the value of $a_{0}(x)=a_{1}(x)=\frac{(1-x)^{2}}{2}=a_{2}(x)=\cdots=a_{n}(x)=\cdots$.

Suppose the solution of Eq. (5.2) has the following form

$$
c(x, t)=c_{0}(x, t)+p c_{1}(x, t) .
$$

Put the values from Eqs. (5.3) and (5.4), when $p=1$ we get the required solution

$$
c(x, t)=\frac{(1-x)^{2}}{2}+\sum_{k=0}^{\infty}\left(\frac{(1-\alpha)^{2} t^{\alpha k}}{\Gamma(\alpha k+1)}-2 \frac{\left(\alpha^{2}-\alpha\right) t^{\alpha k+1}}{\Gamma(\alpha k+2)}+\frac{\alpha^{2} t^{\alpha k+2}}{\Gamma(\alpha k+3)}\right) .
$$

When $\alpha=1$ and tacking $k+2=n$, Eq. (5.5) reduces to

$$
c(x, t)=\frac{(1-x)^{2}}{2}+e^{t}
$$

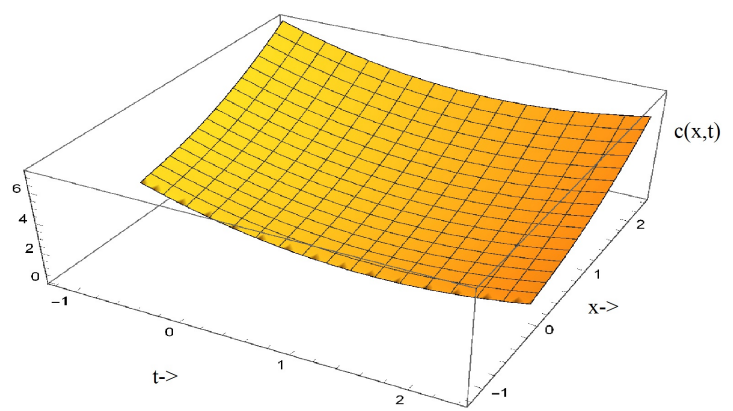

Figure 1: For $\alpha=0.5$

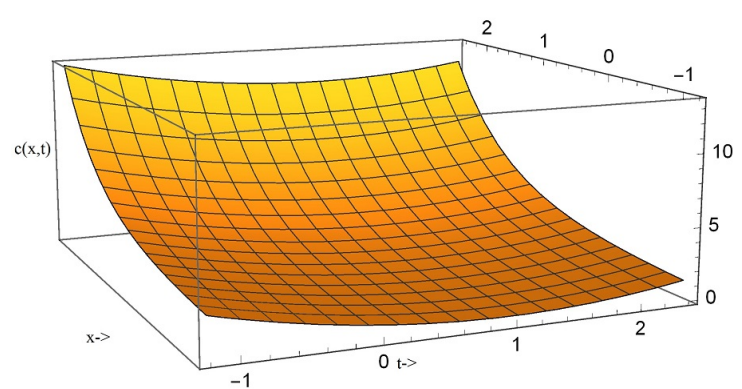

Figure 2: For $\alpha=1$

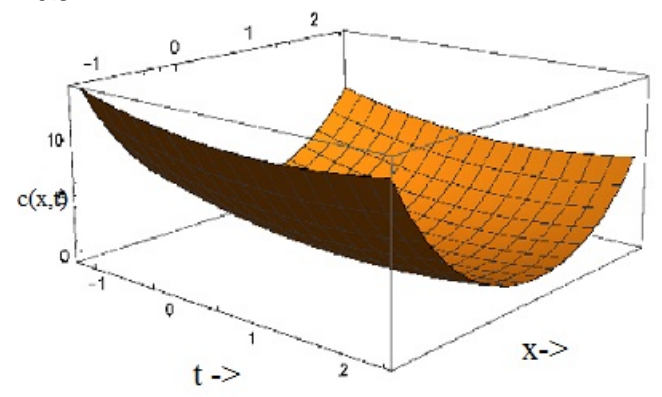

Figure 3: For $\alpha=2$

\section{Conclusions}

In this paper, we have derived the solution of fractional oxygen diffusion equation with the help of LTHPM. Further, we have also discussed the existence and uniqueness of our solution. 


\section{Acknowledgment}

The authors extend their sincere appropriations to the Deanship of Scientific Research at King Saud University for its funding this Profile Research Group (PRG-1437-35).

\section{References}

[1] A. Atangana, On the new fractional derivative and application to nonlinear Fisher's reaction-diffusion equation, Appl. Math. Comput., 273 (2016), 948-956. 1.2, 2

[2] A. Atangana, E. Alabaraoye, Solving a system of fractional partial differential equations arising in the model of HIV infection of $\mathrm{CD}^{+}$cells and attractor one-dimensional Keller-Segel equations, Adv. Difference Equ., 2013 (2013), 14 pages. 1

[3] A. Atangana, B. S. T. Alkahtani, Analysis of the Keller-Segel model with a fractional derivative without singular kernel, Entropy, 17 (2015), 4439-4453. 1

[4] A. Atangana, S. T. A. Badr, Extension of the RLC electrical circuit to fractional derivative without singular kernel, Adv. Mech. Eng., 7 (2015), 1-6. 1

[5] F. B. M. Belgacem, Diffusion and drift models for population dispersal from stochastic and continuum views, Int. J. Appl. Math., 5 (2001), 85-106. 1

[6] M. Caputo, Linear models of dissipation whose Q is almost frequency independent, II, Reprinted from Geophys, J. R. Astr. Soc., 13 (1967), 529-539, Fract. Calc. Appl. Anal., 11 (2008), 4-14. 1

[7] M. Caputo, M. Fabrizio, A new definition of fractional derivative without singular kernel, Progr. Fract. Differ. Appl., 1 (2015), 73-85. 1, 1.1

[8] V. B. L. Chaurasia, R. S. Dubey, Analytical solution for the generalized time-fractional telegraph equation, Fract. Differ. Calc., 3 (2013), 21-29. 1

[9] V. B. L. Chaurasia, R. S. Dubey, F. B. M. Belgacem, Fractional radial diffusion equation analytical solution via Hankel and Sumudu transforms, Int. J. Math. Eng. Sci. Aero., 3 (2012), 1-10. 1

[10] J. Crank, R. S. Gupta, A method for solving moving boundary problems in heat-flow using cubic splines or polynomials, J. Inst. Math. Appl., 10 (1972), 296-304. 1

[11] J. Crank, R. S. Gupta, A moving boundary problem arising from the diffusion of oxygen in absorbing tissue, IMA J. Appl. Math., 10 (1972), 19-23. 1, 1.3

[12] R. S. Dubey, B. S. T. Alkahtani, A. Atangana, Analytical solution of space-time fractional Fokker-Planck equation by homotopy perturbation Sumudu transform method, Math. Probl. Eng., 2014 (2014), 7 pages. 1

[13] R. S. Dubey, P. Goswami, F. B. M. Belgacem, Generalized time-fractional telegraph equation analytical solution by Sumudu and Fourier transforms, J. Fract. Calc. Appl., 5 (2014), 52-58. 1

[14] V. Gülkaç, Comparative study between two numerical methods for oxygen diffusion problem, Comm. Numer. Methods Engrg., 25 (2009), 855-863. 1

[15] A. A. Kilbas, H. M. Srivastava, J. J. Trujillo, Theory and applications of fractional differential equations, North-Holland Mathematics Studies, Elsevier Science B.V., Amsterdam, (2006). 1

[16] A. I. Liapis, G. G. Lipscomb, O. K. Crosser, E. Tsiroyianni-Liapis, A model of oxygen diffusion in absorbing tissue, Math. Modelling, 3 (1982), 83-92. 1

[17] J. Losada, J. J. Nieto, Properties of a new fractional derivative without singular kernel, Progr. Fract. Differ. Appl., 1 (2015), 87-92. 1, 1.1

[18] K. S. Miller, B. Ross, An introduction to the fractional calculus and fractional differential equations, A Wiley-Interscience Publication, John Wiley \& Sons, Inc., New York, (1993). 1

[19] S. L. Mitchell, An accurate application of the integral method applied to the diffusion of oxygen in absorbing tissue, Appl. Math. Model., 38 (2014), 4396-4408. 1, 1.3

[20] B. Noble, Heat balance methods in melting problems, J. R. Ockendon, W. R. Hodgkins (Eds.), Moving boundary problems in heat flow and diffusion, Clarendon Press, Oxford, (1975), 208-209. 1

[21] I. Podlubny, Fractional differential equations, An introduction to fractional derivatives, fractional differential equations, to methods of their solution and some of their applications, Mathematics in Science and Engineering, Academic Press, Inc., San Diego, CA, (1999). 1

[22] W. C. Reynolds, T. A. Dolton, The use of integral methods in transient heat transfer analysis, ASME paper, (1958). 1 